\title{
VAST Clinical Trial: Safely Supplementing Tissue Lost to Degenerative Disc Disease
}

\author{
DOUGLAS P. BEALL, MD, ${ }^{1}$ GREGORY L. WILSON, DO ${ }^{2}$ RANDOLPH BISHOP, MD, ${ }^{3}$ \\ WILLIAM TALLY, $\mathrm{MD}^{4}$ \\ ${ }^{1}$ Summit Medical Center, Edmond, Oklahoma, ${ }^{2}$ Invictus Healthcare, Tulsa, Oklahoma, ${ }^{3}$ Neurological and Spine Institute, Savannah, Georgia, \\ ${ }^{4}$ Athens Orthopaedic Clinic, Athens, Georgia
}

\begin{abstract}
Background: The function of the intervertebral disc is structural. Loss of tissue alters biomechanics, leads to subsequent disc degeneration, and is attributable to discogenic pain. A viable structural allograft was delivered into degenerate discs to determine whether intervention could safely stabilize anatomy, reduce pain, and improve function.

Methods: Following institutional review board approval and patient consent, subjects were randomized to receive allograft or saline at either 1 or 2 levels or continue nonsurgical management (NSM). Data were collected at baseline, 3 , 6, and 12 months. Back pain with a visual analog scale (VAS) and disability by the Oswestry Disability Index (ODI) were assessed, as were adverse events. This trial is registered on http://www.clinicaltrials.gov (NCT03709901).

Results: At 6 and 12 months, the VAS improved from 54.81, 55.25, and 62.255 in the allograft, saline, and NSM subjects, respectively, to 16.0 and 41.0 in the allograft and saline groups at 6 months, and 12.27 and 19.67, respectively, at 12 months. All subjects in the NSM cohort crossed over to allograft treatment. At 6 and 12 months, ODI improved from 53.73, 49.25, and 55.75 in the allograft, saline, and NSM subjects, respectively, to 18.47 and 28.75 in the allograft and saline groups 1 and 2 at 6 months, and 15.67 and 9.33, respectively, at 12 months. At 3 months the ODI of the NSM group was 62.75 and subjects reached 19.0 and 11.0 at 6 and 12 months, respectively. Adverse events were transient and resolved in all cohorts.

Conclusions: This study is supported by data demonstrating that improved pain and function at 12 months can be attained with a supplemental viable disc matrix. Subjects receiving the VIA Disc Matrix achieved improvements that were durable at 12 months.

Level of Evidence: 1.

Clinical Relevance: Initial assessments indicate that a 1-level or 2-level treatment offers a reliable intervention that is safe and beneficial.

Biologics

Keywords: disc degeneration, viable disc matrix, supplemental disc allograft, back pain
\end{abstract}

\section{INTRODUCTION}

Vertebrates arose more than 530 million years ago, and the evolution of vertebral bone from the flexible notochord was a key event that provided foundation morphology supporting a structural anatomy that renders the spine stiffer while bending. This attribute of bending without shortening contributes to important mechanical features that enable storing and releasing energy elastically like a spring.

The function of the intervertebral disc is predominantly structural, supporting upright posture without compromising freedom of rotation, flexion, or compression. With such degrees of freedom, it is the perfect tissue to support bipedal anatomy and the active use of upper extremities while standing or ambulating. The advent of flexibility comes with a parallel morphology that is unique to the intervertebral disc, which contains structured water that carries hydraulic qualities, and mechanically plastic properties that simultaneously support movement and resistance to stress. Retaining the notochordal qualities centrally as a hallmark of elasticity in the nucleus pulposus, the evolutionary stiffening of the vertebral bodies created a flexible load bearing structure that has unfortunately made the human spine particularly prone to pathology.

A high concentration of proteoglycans in the nucleus pulposus produces an intradiscal pressure that is imperative to the mechanical function of the disc. Unfortunately for sustaining long-term function, the disc has a low capacity for replenishment. 
It is alymphatic, aneural, and avascular; therefore, it is dependent on transfer of nutrients to and metabolic waste across the subchondral plate. Over time, degeneration takes its toll on the structure, with a loss of proteoglycans leading to a loss of intradiscal pressure due to the decreasing ability to hold structured water and the loss of functional tissue and altered mechanics. Over time the tissue loss and resultant mechanical alteration of the disc renders the intervertebral disc unstable. The inherent degrees of freedom that are assets to disc mechanics in healthy tissue are liabilities under degenerate conditions. Laxity of the spine can produce spondylolisthesis and reduce disc height; pain emerges as a result of this instability and abnormal force transmission.

Low back pain is the leading cause of disability in developed countries, with the number of people affected worldwide increasing annually. ${ }^{1}$ Degenerative disc disease is a major factor contributing to this disability and is the most common etiology of chronic lower back pain in adults. More than 40\% of the adult population of the United States reported low back pain at some point within the preceding 3 months prior to them being questioned and this has a substantial socioeconomic impact on the affected population. ${ }^{2,3}$ Causal factors vary considerably from the innocuous insult of repeated heavy lifting or sudden awkward movement resulting in an acute injury to the more insidious condition of disc degeneration. Though several risk factors have been identified (including occupational posture, depressive moods, obesity, body height, and age), the genesis of low back pain remains obscure. Back pain is not a disease but a constellation of symptoms, and in most cases the origins remain unknown.

Low back pain affects people of all ages, from children to the elderly. Estimating the initial back pain incidence is challenging due to a relatively high prevalence of back pain in early adulthood that persists with recurrent symptoms over time. That noted, the lifetime prevalence of nonspecific (common) low back pain has been estimated at $60 \%$ to $70 \%$ in industrialized countries (1-year prevalence, $15 \%$ to $45 \%$; adult incidence, $5 \%$ per year), with prevalence and incidence peaking between the ages of 35 and 55 years. ${ }^{4,5}$ As the world population gets older, low back pain is expected to increase substantially as an inevitable consequence of aging. Low back and neck pain in the United States was the third-largest condition of spending in 2013, with estimated health care spending of $\$ 87.6$ billion. $^{6}$ In recent years, there has been a substantial increase in health care costs to treat low back pain, and most of these treatments are simply to provide symptomatic relief with few strategies that actually contribute to correcting the underlying cause. ${ }^{7}$ Examples of these treatments are multiple but consistently embrace strategies that relieve symptoms before considering treatments designed to retard further degeneration. Lower back pain prevalence and social impact is further complicated by the growing levels of obesity and sedentary lifestyles, which are strongly associated with low back pain. ${ }^{8}$

Treatment for degenerative disc disease typically starts with nonsurgical methods that are intended to provide sufficient symptomatic relief by reducing pain from the damaged disc. Designated as conservative care, or nonsurgical management (NSM), methods of pain control may include anti-inflammatory medications, manual manipulation, steroid injections, electrical stimulation, back braces, and heat or ice therapy. In concert with treatments intended to provide symptomatic relief, other therapies such as physical therapy or yoga that restore functional mechanics are widely endorsed. Physical therapy provides an avenue for stretching and strengthening muscles that helps the lumbar spine heal and reduces the frequency of painful flare-ups. Several years ago, the North American Spine Society recommended postoperative physical therapy after surgery for degenerative conditions of the lumbar spine., ${ }^{9,10}$ Lifestyle modifications such as correcting posture, losing weight, or giving up smoking can sometimes help reduce stress on and improve healing of the damaged disc and can potentially slow down further degeneration.

In the end, structural degeneration changes loading and exacerbates both the mechanical and biologic-pathologic processes. These altered structural changes affect adjacent vertebrae and lead to facet joint arthritis, bone spur, or osteophyte formation and contribute to spine diseases such as canal stenosis and spondylolisthesis (Figure 1). The Thompson depiction of degeneration represents one of the first attempts to classify stages of disc degeneration, but many grading systems have been developed subsequently. ${ }^{11,12}$

Intradiscal tissue loss resulting from disc degeneration is implicated as a factor in mechanical imbalance that, coupled with subsequent matrix 


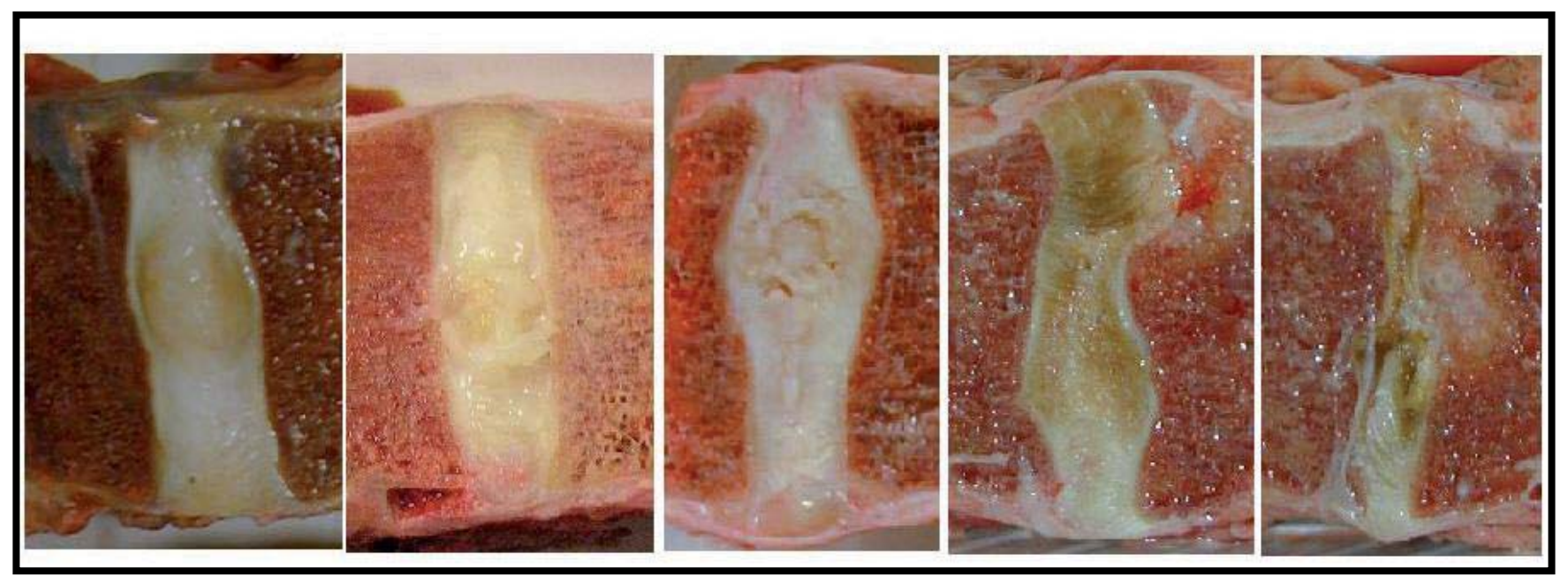

\section{Synthesis}

Figure 1. Disc degeneration is a progressive imbalance of anabolic and catabolic processes that result in tissue loss, which disrupts the anatomy, alters the loading, and results in progressive desiccation. Depiction of disc degeneration based on Thompson Grading. ${ }^{11}$

loss, drives a cyclic process that gives rise to further instability and additional disc degeneration. This cycle of degeneration is repetitive and often progressive.

Whereas the exact origin of intervertebral disc degeneration remains to be identified, changes centered in the nucleus pulposus are believed to be the origin of the degenerative changes because the tissue in this region displays the severest change during the early stages of intervertebral disc degeneration. ${ }^{13}$ The variation in tissue composition during the degenerative process compromises the structural integrity, which further alters the metabolic activity and biochemical function within the disc.

The most common biochemical characteristics of intervertebral disc degeneration are the loss of glycosaminoglycan and the accompanying decrease of water content. These changes have been attributed to nutritional deprivation and inappropriate mechanical loading and are further linked to genetic, metabolic, and mechanical imbalances. ${ }^{14-20}$

How each of these causal factors leads to the various types of disc degeneration patterns and degeneration progression remains incompletely known despite the fact that variations in disc chemistry have been widely published and presented over the past 30 years. $^{21}$ The critical remedy for intervertebral disc degeneration has yet to emerge as a structured solution for progressive disc degeneration. ${ }^{2-24}$ As the largest avascular structure in the human body, the intervertebral disc balances dual roles that require mechanical support without sacrificing spine flexibility. The uncertain origin of disc degeneration makes it difficult to determine whether matrix loss or mechanical perturbation is the primary insult to tissue homeostasis. The tissue integrity of the disc is further complicated by the lack of vascular, lymphatic, and direct neural integration directly to the central disc anatomy, and interventions intended for restoration require direct placement.

The development of chronic back pain in conjunction with degenerative disc disease often results in surgical treatment. Besides nonsurgical methods such as rest, physical therapy, and bracing, surgical procedures such as discectomy and placement of a total intervertebral disc prosthesis as well as replacement of the nucleus are now used for treatment of chronic back pain after disc herniation. ${ }^{25-31}$ So far, no clinically established procedure is available that slows the progression of intervertebral disc degeneration. In this context, the question has been raised about the ability of the 
intervertebral disc to regenerate its cellular matrix subsequent to either degenerative loss of tissue or discectomy.

Clearly, any reparative approach intended to facilitate motion preservation has to recognize that biomechanical stability and matrix composition are intrinsically connected. A strategy to retard or restore intervertebral disc tissue must initially stabilize the mechanical imbalance while at the same time supplement the disc matrix loss. The current unmet clinical need demonstrates that solutions to date fall short of achieving regenerative results and that beyond palliative relief, the clinical solution to the problem is essentially unsatisfactory.

Various biological techniques have been developed and tested to treat the degenerative intervertebral disc. Common to each has been the aim to sustain delivery of biologically active factors to the disc that might guide regeneration or at least retain the status quo of the affected tissue and retard further regeneration. General approaches that have been advance over many years include

1. Delivery of a growth factor, or other singular novel factor by intradiscal injection ${ }^{32,33}$

2. Gene therapy approaches that modify gene expression of resident disc cells in vivo (direct gene therapy) $)^{34,35}$

3. Autologous implantation of cells that have been antecedently removed, cultivated, and often modified in vitro ${ }^{36-41}$

4. Implantation of mesenchymal stem cells of a variety of in vitro-derived cell lineages has been proven in animal models. ${ }^{42-44}$

Using biologics heralds a new optimism that pain relief and functional improvement might be possible, but they lack attention to the structural deficit that results from disc degeneration. The structural deficit may be primary or secondary, but the difference is largely academic if the primary cause remains unknown. Supplementation of the disc addressing both biologic and tissue replenishment simultaneously might afford a distinct advantage in treating intervertebral disc degeneration.

This clinical study was developed to evaluate a technology that provides the benefits of allograft supplementation combined with a matrix replete with viable cells. Variations of porosity and cell density have been shown to affect the physical properties, such as the swelling ratio, stiffness, and mechanical strength, and theoretical reports have defined an optimal cell number that will produce the greatest disc repair. ${ }^{45,46}$ Replacing lost tissue or at least attempting to subsidize biomechanical components may overcome a loading imbalance resulting from tissue loss. Using a viable allograft matrix with cells that are anatomically appropriate for the spine is a second step, and being able to deliver the supplemental graft to the nucleus pulposus without causing undue additional damage to the annulus is the final necessary step.

The study was also conducted to evaluate whether a product developed for allograft supplementation could be safely administered and whether a formulation designed to replace tissue lost to degenerative disc changes might be presumably physiologic as well. There is no shortage of studies that have successfully transplanted cells into discs or nucleus pulposus material into disc, but no clinical applications that bring together the advantages of each have been tested.

\section{MATERIALS AND METHODS}

\section{Clinical Study Design}

The Viable Allograft Supplemented Disc Regeneration in the Treatment of Patients with Low Back Pain With or Without Disc Herniation (VAST) Trial was conducted, subjects enrolled, and data gathered under jurisdiction and oversight from the Sterling Institutional Review Board (Atlanta, GA) from February 2017 to August 2019. The subject evaluation has been extended to 24 and 36 months. The VAST Trial is registered at ClinicalTrials.gov (Identifier: NCT03709901). ${ }^{47}$

The VAST Trial was a prospective, randomized, parallel-arm, multicenter study approved to enroll up to 220 subjects at up to 15 clinical sites. Outcomes of the trial were based on assessment of primary and secondary endpoints 6 and 12 months after transplant of supplementary allograft compared with placebo or sustained conservative care (NSM) in subjects who have discogenic pain attributable to disc degeneration as judged by MRI scoring (Pfirrmann), physical examination, and subject-reported pain.

The study consisted of 2 phases: a screening phase (enrollment) followed by the active phase (12 months). As outlined (Figure 2), there was an indeterminate overlap of up to 2 weeks between the end of the screening phase and the start of the 


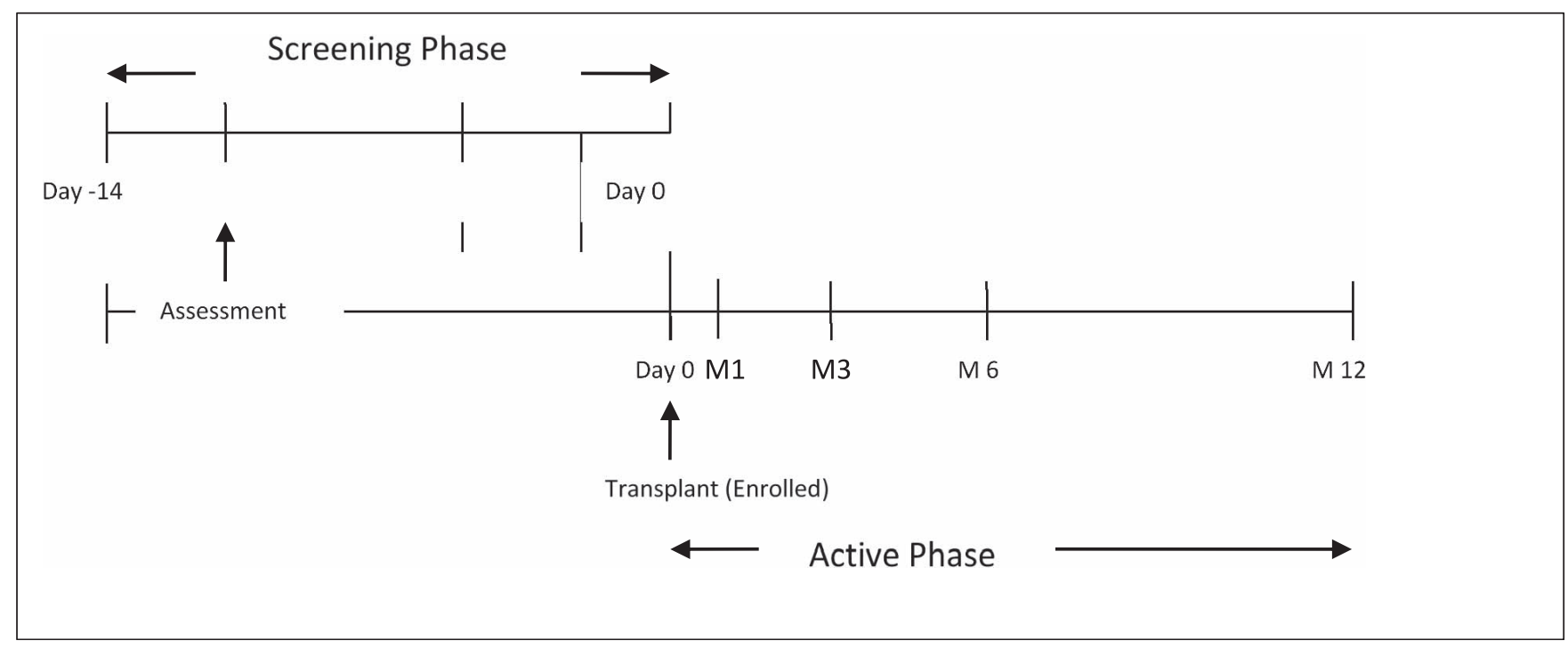

Figure 2. The study consisted of 2 phases: a screening phase (enrollment) followed by the active phase (12 months). As outlined, there was an indeterminate overlap of up to 2 weeks between the end of the screening phase and the start of the active phase for individual subjects. Subjects met entry criteria for both phases to be eligible and 2 weeks' opportunity was allowed for the subjects to evaluate the protocol and consent to their participation. Although 2 weeks were provided, subjects were eligible following their consent documentation being reviewed and received.

active phase for individual subjects. Subjects met entry criteria for both phases to be eligible and 2 weeks' opportunity was allowed for the subjects to evaluate the protocol and consent to their participation. Although 2 weeks were provided, subjects were eligible following their consent documentation being reviewed and received.

The aim of the VAST Trial was to investigate the clinical relevance of treating painful intervertebral disc tissue by a supplementary transplantation of viable cellular allograft disc matrix in a controlled clinical study comparing the cellular allograft with a saline placebo or continued treatment with NSM. Clinical differences among the 3 groups were compared by analyzing pain, functional restrictions, and neurological deficits using different scores that were especially developed for the assessment of degenerative spine complaints. ${ }^{48}$ In addition, radiographic evaluations and MRI were performed to evaluate morphologic changes that might distinguish differences between the 2 treatment groups. Radiographic evaluations and MRI included changes in the height of the intervertebral disc, Modic changes of the adjacent endplates, and the fluid content of the operated intervertebral disc. ${ }^{49,50}$ The target strategy of this new product is to slow down the complex degeneration process of the intervertebral disc and to offer a prescriptive option to therapeutic intervention that is clinically relevant and economically efficient. ${ }^{51}$

\section{Subjects}

The study population in this report consisted of 24 subjects of various ethnicities from 18 to 60 years of age who demonstrated clinical disc degeneration at 1 or 2 vertebral levels from L1 to S1 (Figure 3). Patients were classified with moderate to severe disability (ODI $\geq 40 \%$ ) and pain (VAS $\geq 40 \mathrm{~mm}$ ) that was chronic during the screening Phase and demonstrated modified Pfirrmann levels 3 to 6 on MRI. Subjects were included who demonstrated type 1 or type 2 Modic changes. Eligible subjects had experienced chronic low back pain for a minimum of 6 months, had no contraindications to allograft transplantation, and were willing to return to the clinic for multiple safety and efficacy assessments for up to 12 months following enrollment.

Primary exclusion criteria included radicular pain, symptomatic spinal stenosis, disc protrusion $>5 \mathrm{~mm}$, spondylolisthesis $>5 \mathrm{~mm}$ at any level, and body mass index $>35$. Detailed inclusion and exclusion criteria are presented in Table 1. Subjects participating in the VAST Trial were randomized at 3.5:1:1 to receive either a supplemental allograft (VIA Disc Matrix), saline as a placebo, or to continue under NSM. The assignment of the subjects to treatment (viable matrix supplement, saline placebo, or standard of care) was performed in a randomized manner after informed consent was obtained. Upon confirmation of eligibility, baseline measurements were collected and retained at individual study sites, and clinical data were monitored 


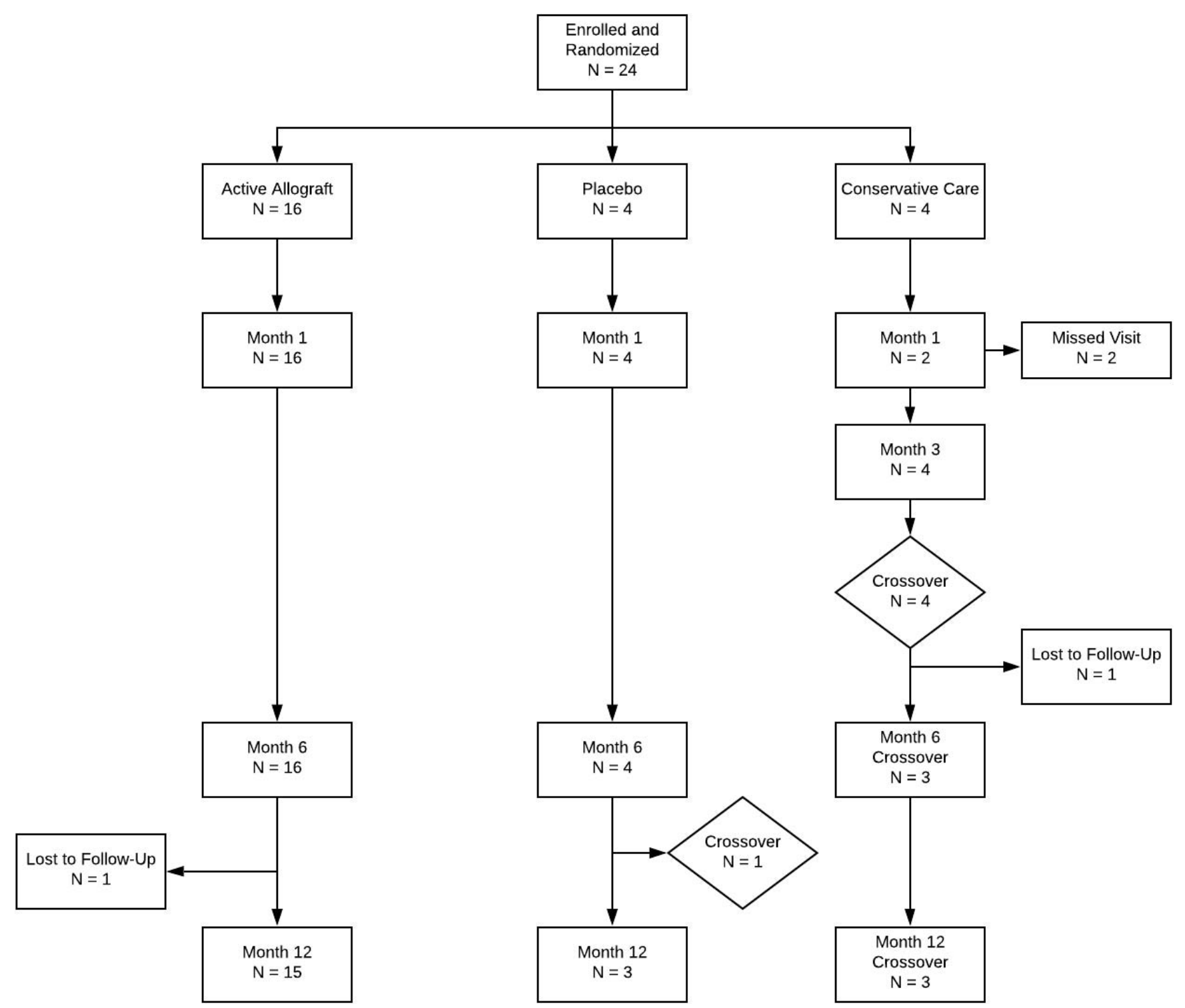

Figure 3. Subjects participating in the VAST Trial were randomized at 3.5:1:1 to receive a supplemental allograft (VIA Disc Matrix), saline as a placebo, or to continue under nonsurgical management (NSM). The assignment of the subjects to treatment (viable matrix supplement, saline placebo, or standard of care) was performed in a randomized manner after informed consent has been obtained.

independently and entered by a third party (MileStone CRO, San Diego, CA).

To evaluate and minimize subject risk, the first 24 subjects (at least 4 from each group) were evaluated 1 month after transplantation for a full safety assessment including radiographic and MRI information. All data collected were reviewed by the steering committee, who determined whether recruitment of patients as subjects could continue. The parameters used by the safety committee to determine whether recruitment resumed included the following:

1. A comparison of the adverse event (AE) profile between the 2 groups including rate, relationship, and severity.
2. A comparison of the clinical laboratory parameters between the 2 groups

3. A comparison of vital signs and physical examination findings between the 2 groups

4. A comparison of radiographic and MRI findings between the 2 groups

The first 24 subjects selected to participate in the study returned to the clinic for a safety assessment 1 month after their transplant. Subsequent clinic visits occurred at 6 and at 12 months following the transplant for safety and efficacy assessments. Subjects randomized to NSM were similarly followed to ensure no compromise in their care, and they additionally received a clinical follow-up by 
Table 1. Inclusion and exclusion criteria.

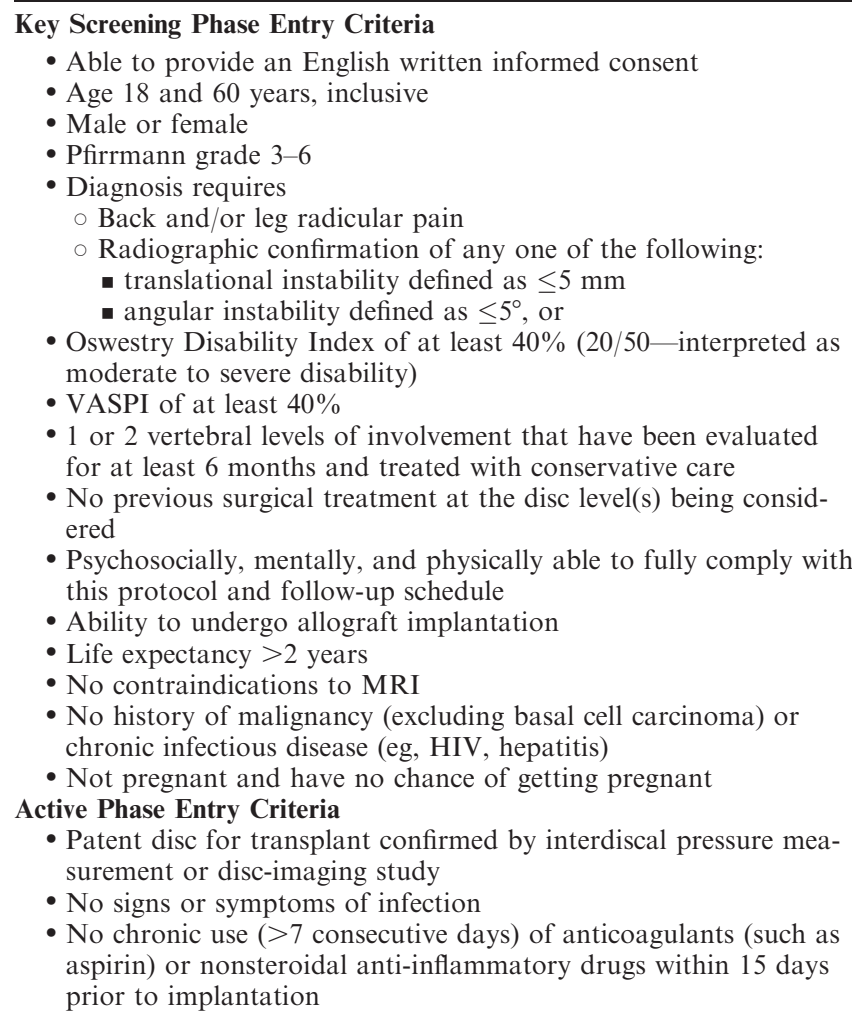

phone at 3 months to assess pain level and loss of function since their initial visit. Those subjects who felt they were not improving were offered VIA Disc Matrix and followed for an additional 12 months from the index procedure (implantation of the supplemental graft).

\section{Study Interventions}

Subjects screened, eligible, and randomized to the active phase of the study underwent a transplantation procedure. This procedure was performed under fluoroscopic guidance with moderate conscious sedation at the investigator's discretion in an outpatient setting. Subjects receiving the active treatment were treated identically to the point of the material preparation and the treatment placed. Subjects were not told of their treatment, and although a more detailed procedure for preparing the graft was required, subjects were not able to compare that preparation time with that of saline.

The preserved cell vial and mixing device were removed from the outer packaging using standard aseptic technique, presenting the cell vial and mixing device to the operative field. After the contents of the cell vial were completely thawed, the cell solution and nucleus pulposus allograft and saline components were mixed thoroughly.

Subjects were placed in the prone position on a multiplanar fluoroscopy table, propped, and bolstered for patient comfort and positioning for the procedure. Preliminary fluoroscopy was used to localize the target and mark the vertebral bodies and the disc(s) to be implanted. The skin was cleaned with betadine, and the subject was draped. Skin and deeper tissues were infiltrated with local anesthetic. A 22-gauge, 5-in spinal needle was inserted through Kambin's triangle at the index disc level(s) to be injected, care taken to avoid visceral, vascular, or neural injury. Placement of the needle was confirmed on additional imaging (anteroposterior and lateral fluoroscopic imaging, computed tomography [CT], CT fluoroscopy).

After determination that the needle tip was in an optimal location, the allograft was connected to the 22-gauge needle and 1.25 to $1.75 \mathrm{~cm}^{3}$ of the allograft was injected into the affected disc under real-time fluoroscopic imaging. Subjects were transferred to the recovery room for monitoring. The techniques for active transplant varied only in preparation of the material:

1. Supplemental viable allograft (VIA Disc Matrix) was prepared from human nucleus pulposus allograft that contains allogeneic viable cells. Each allogeneic product was individually prepared, but not specific for the subject being treated. A minimum of $6 \times 10^{6}$ cells were suspended in allograft matrix suspension.

2. Each subject who received the saline placebo was similarly positioned, the needle tip placement was identified, and the subject received approximately $1.75 \mathrm{~cm}^{3}$ per level as a placebo treatment.

\section{Data Management}

Data concerning the outcome of the supplemental transplant and the demographic data were collected during the visits and documented on appropriate case report forms. For all visits and for each patient, the case report forms were completed and all results uploaded by MileStone for data management and analysis after source data verification by the responsible monitor had been completed. 


\section{Interim Analysis}

For safety and efficacy reasons, interim analyses performed after the first 24 subjects had completed the month 1 assessment of the active phase were done in a descriptive manner only and no unblinding occurred. A steering committee convened to review the results of the interim analysis and reviewed 6-month and 12-month data sets for VAS, ODI, and AEs.

\section{Statistical Methods and Determination of Sample Size}

The statistical evaluation was done with appropriate statistical software packages. For all baseline data, the descriptive statistics were presented. In the case of continuous parameters, statistical parameters for location and dispersion (eg, n, mean, standard deviation, range) were calculated. For discrete data, appropriate frequency tables were produced.

\section{Primary Criteria}

The primary criteria for the entire population were analyzed using a 2-sided approach on an $\alpha$ level of .05 based on the total score. A hierarchical test procedure was applied. In the first step the prepost difference of the groups was compared using the Wilcoxon rank sum test. When there was a significant result, a responder analysis was done; the analysis and results that followed spoke to the first 24-subject safety cohort but lacked adequate power for statistical analysis. Secondary variables were analyzed in an exploratory way using adequate statistical procedures to include the powered groups.

\section{RESULTS \\ Efficacy and Safety Measurements}

Per the study protocol, interim analysis of the first 24 subjects enrolled was undertaken at the 12month follow-up, which mirrors the primary endpoint of the complete subject enrollment and analysis. An independent analysis and safety audit were carried out in conjunction with the data monitoring and review (Boston Biomedical Associates, Boston, MA). Demographics and baseline characteristics were similar among the 3 groups after randomization (Table 2).

\section{Efficacy and Safety Measurements}

There were 204 subjects at 13 US sites enrolled in the VAST Trial. These individuals were segmented into a treatment group, an NSM group, and a saline placebo control group with a 3.5:1:1 randomization ratio. The first 24 participants were assessed at 1month posttreatment to assess for safety. There were two coprimary endpoints including back pain as measured by the VAS and function as measured by the ODI. The primary endpoints were evaluated along with safety data and reported AEs and changes in clinical laboratory evaluations. The data were collected at baseline and at 3, 6, and 12 months. Structural evaluation was also performed and imaging studies including x-rays and MRI were performed at 6 and at 12 months.

Adverse events and serious AEs were evaluated in the 24-subject safety cohort. There were 13 events reported, 12 in the active allograft and 1 in the saline placebo. Events in the NSM cohort did not report any events associated with treatment even following crossover. Only 1 of the AEs was possibly associated with treatment. All events resolved, and none of the subjects left the trial. No serious AES were reported, and 6 of the 16 subjects accounted for the 12 AEs in the active allograft. Within the saline placebo group, 1 of the 4 reported an AE, and an additional subject who randomized to the saline placebo arm did not obtain meaningful pain relief and was provided the allograft at 6 months. In retrospect, 1 of $3(33 \%)$ of the subjects randomized to placebo was affected, and 6 of $16(37.5 \%)$ of those subjects randomized and blinded to the VIA Disc Matrix allograft were affected. The chief concern most often reported was back pain, and more than $50 \%$ of the AEs were generated from 3 of 16 subjects in the allograft cohort (Table 3 ).

\section{Visual Analog Scale, Oswestry Disability Index}

The data on the first 24 patients at 12-month follow-up were noted at the 6- and 12-month time points (Figures 4 and 5). The VAS back pain improved from 54.81, 55.25, and 62.25 in the allograft, placebo, and NSM subjects, respectively, to $16.0,41.0$, and 6.67 at 6 months and to 12.27 , 19.67, and 6.0 at 12 months (Figure 4). At 3 months the VAS of the NSM group was 54.0. There was an option for the NSM patients to cross over to the allograft treatment group at the 3month time point, and all subjects elected to cross 
Table 2. Demographics and baseline characteristics safety cohort.

\begin{tabular}{|c|c|c|c|}
\hline & Active Allograft, $n=16$ & Placebo, $n=4$ & Conservative Care, $n=4$ \\
\hline \multicolumn{4}{|l|}{ Age, y } \\
\hline Mean \pm SD (n) & $38.25 \pm 8.68(16)$ & $44.50 \pm 12.12(4)$ & $40.50 \pm 5.32(4)$ \\
\hline Median (min, $\max$ ) & $37.00(27.00,62.00)$ & $40.50(35.00,62.00)$ & $40.50(34.00,47.00)$ \\
\hline \multicolumn{4}{|l|}{ Gender } \\
\hline Female, \% (n) & $12.5(2)$ & $0.0(0)$ & $50.0(2)$ \\
\hline Male, \% (n) & 87.5 (14) & $100.0(4)$ & $50.0(2)$ \\
\hline \multicolumn{4}{|l|}{ Race } \\
\hline White, \% (n) & $87.5(14)$ & $100.0(4)$ & $75.0(3)$ \\
\hline White/American Indian, \% (n) & $6.3(1)$ & $0.0(0)$ & $0.0(0)$ \\
\hline American Indian, \% (n) & $6.3(1)$ & $0.0(0)$ & $25.0(1)$ \\
\hline \multicolumn{4}{|l|}{ Ethnicity } \\
\hline Hispanic, \% (n) & $6.3(1)$ & $0.0(0)$ & $0.0(0)$ \\
\hline Non-Hispanic, \% (n) & $93.8(15)$ & $100.0(4)$ & $100.0(4)$ \\
\hline \multicolumn{4}{|l|}{ Body mass index, $\mathrm{kg} / \mathrm{m}^{2}$} \\
\hline Mean \pm SD (n) & $25.66 \pm 3.21(16)$ & $29.65 \pm 3.09$ (4) & $29.80 \pm 8.27(4)$ \\
\hline Median (min, max) & $24.40(21.90,32.30)$ & $30.85(25.10,31.80)$ & $33.05(17.70,35.40)$ \\
\hline \multicolumn{4}{|l|}{ Smoking history } \\
\hline Never, \% (n) & $75.0(12)$ & $50.0(2)$ & $75.0(3)$ \\
\hline Past smoker, \% (n) & $18.8(3)$ & $50.0(2)$ & $25.0(1)$ \\
\hline Current smoker, \% (n) & $6.3(1)$ & $0.0(0)$ & $0.0(0)$ \\
\hline \multicolumn{4}{|c|}{ History of endocrine or metabolic disorders } \\
\hline Yes, \% (n) & $0.0(0)$ & $25.0(1)$ & $0.0(0)$ \\
\hline No, $\%(n)$ & $100.0(16)$ & $75.0(3)$ & $100.0(4)$ \\
\hline \multicolumn{4}{|l|}{ Levels of treatment } \\
\hline One level, \% (n) & $68.8(11)$ & $50.0(2)$ & $50.0(2)$ \\
\hline Two levels, \% (n) & $31.3(5)$ & $50.0(2)$ & $50.0(2)$ \\
\hline
\end{tabular}

over to allograft treatment. At the 6- and 12-month time points the ODI improved from 53.73, 49.25, and 55.75 in the allograft, placebo, and NSM subjects, respectively, to $18.47,28.75$, and 19.0 at 6 months, and to $15.67,9.33$, and 11.0 at 12 months (Figure 5). At 3 months the ODI of the NSM group was 62.75 and all subjects crossed over to allograft treatment. MRI evaluation showed anatomic improvement of the disc and enhanced nucleus signal (Figures 6 and 7).

Data from the first 24 subjects as part of this large triple-arm prospective randomized control trial showed that a delivery of a viable structural allograft can be done safely with no AEs related to the procedures in these initial subjects followed up to 1 year. Subjects receiving the allograft had a larger reduction of pain and a greater functional improvement than did the placebo and NSM cohorts, and those NSM subjects crossing over to allograft supplementation attained similar pain and functional improvements to those initially randomized to receive the active treatment (Figures 4 and 5). The safety data were not powered for statistical significance, but the prominent improvements in pain and function trend toward the possibility of statistically significant differences at the final analysis of the data.

Table 3. Study cohort demographics.

\begin{tabular}{|c|c|c|c|c|c|c|}
\hline & \multicolumn{2}{|c|}{ Active Allograft, $n=16$} & \multicolumn{2}{|c|}{ Placebo, $n=4$} & \multicolumn{2}{|c|}{ Conservative Care, $n=4$} \\
\hline & $\begin{array}{l}\text { No. of } \\
\text { Events }\end{array}$ & $\begin{array}{c}\text { No. of } \\
\text { Subjects, \% (n) }\end{array}$ & $\begin{array}{l}\text { No. of } \\
\text { Events }\end{array}$ & $\begin{array}{c}\text { No. of } \\
\text { Subjects, \% (n) }\end{array}$ & $\begin{array}{l}\text { No. of } \\
\text { Events }\end{array}$ & $\begin{array}{c}\text { Total No. of } \\
\text { Subjects, \% (n) }\end{array}$ \\
\hline Total number of AEs & 12 & $37.5(6)$ & 1 & $25.0(1)$ & 0 & $0.0(0)$ \\
\hline Injury, poisoning and procedural complications & 1 & $6.3(1)$ & 0 & $0.0(0)$ & 0 & $0.0(0)$ \\
\hline Muscle injury & 1 & $6.3(1)$ & 0 & $0.0(0)$ & 0 & $0.0(0)$ \\
\hline Investigations & 1 & $6.3(1)$ & 0 & $0.0(0)$ & 0 & $0.0(0)$ \\
\hline Red blood cell sedimentation rate increased & 1 & $6.3(1)$ & 0 & $0.0(0)$ & 0 & $0.0(0)$ \\
\hline Musculoskeletal and connective tissue disorders & 9 & $25.0(4)$ & 1 & $25.0(1)$ & 0 & $0.0(0)$ \\
\hline Arthralgia & 0 & $0.0(0)$ & 1 & $25.0(1)$ & 0 & $0.0(0)$ \\
\hline Back pain & 7 & $18.8(3)$ & 0 & $0.0(0)$ & 0 & $0.0(0)$ \\
\hline Musculoskeletal pain & 1 & $6.3(1)$ & 0 & $0.0(0)$ & 0 & $0.0(0)$ \\
\hline Pain in extremity & 1 & $6.3(1)$ & 0 & $0.0(0)$ & 0 & $0.0(0)$ \\
\hline Nervous system disorders & 1 & $6.3(1)$ & 0 & $0.0(0)$ & 0 & $0.0(0)$ \\
\hline Burning sensation & 1 & $6.3(1)$ & 0 & $0.0(0)$ & 0 & $0.0(0)$ \\
\hline
\end{tabular}

Abbreviation: AE, adverse event. 


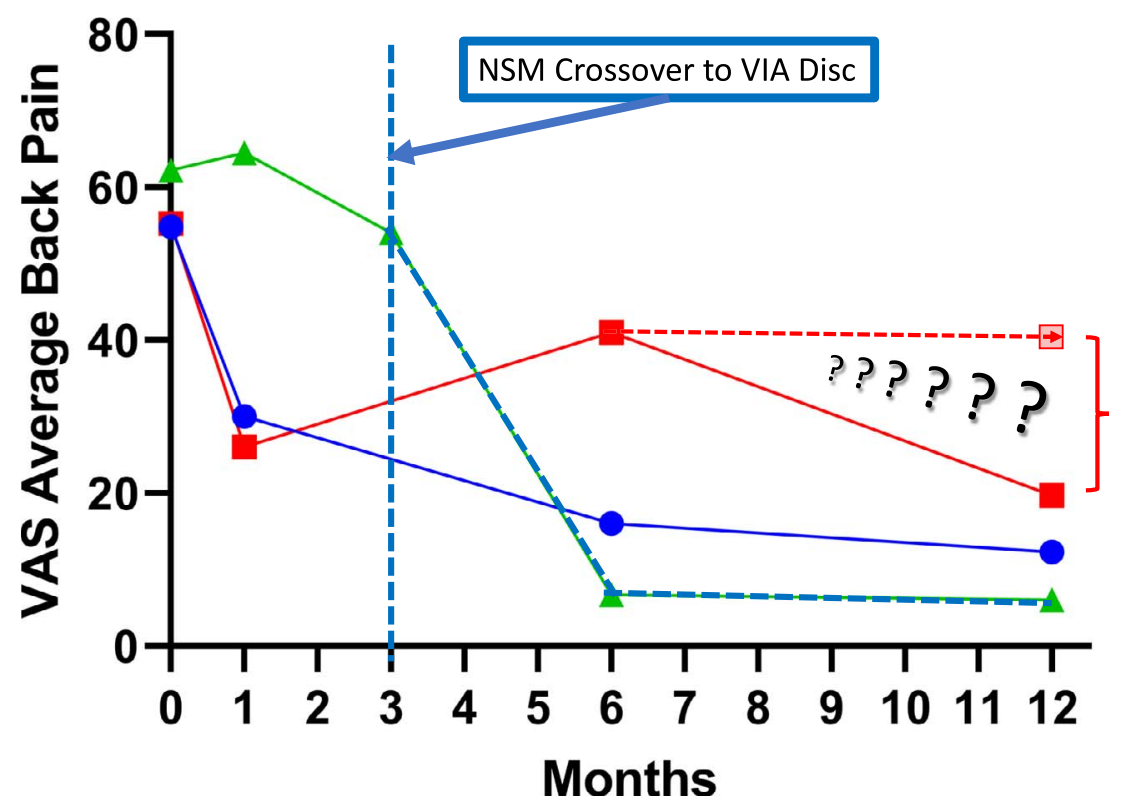

\section{- Active Allograft \\ - Placebo}

$\rightarrow$ Conservative Care

A Placebo Subject Dropped out after 6 months and the trajectory in the small group was skewed. Dotted Line reflects a difference if that subject had been evaluated at 12 months

NSM Crossover

Figure 4. Reduction in pain was evident in both of the active treatment groups in the study. Subjects who had been randomized to continue nonsurgical management (NSM) demonstrated no relief. The NSM subjects were further evaluated at 3 months (vertical dashed line), and unable to continue, all subjects crossed over to VIA Disc Tissue Matrix. Their response followed the trajectory of the active arms of the study and outcome demonstrated the largest improvement. One subject in the saline-treated group could not continue the study, and after 6 months crossed over to VIA Disc. The horizontal dashed line reflects that progress averaged into the small (4 subjects) placebo group. All patients receiving the randomized allograft completed the study in their cohort. In total, 21 of 24 subjects received the VIA Disc Tissue Matrix either randomized or open label.

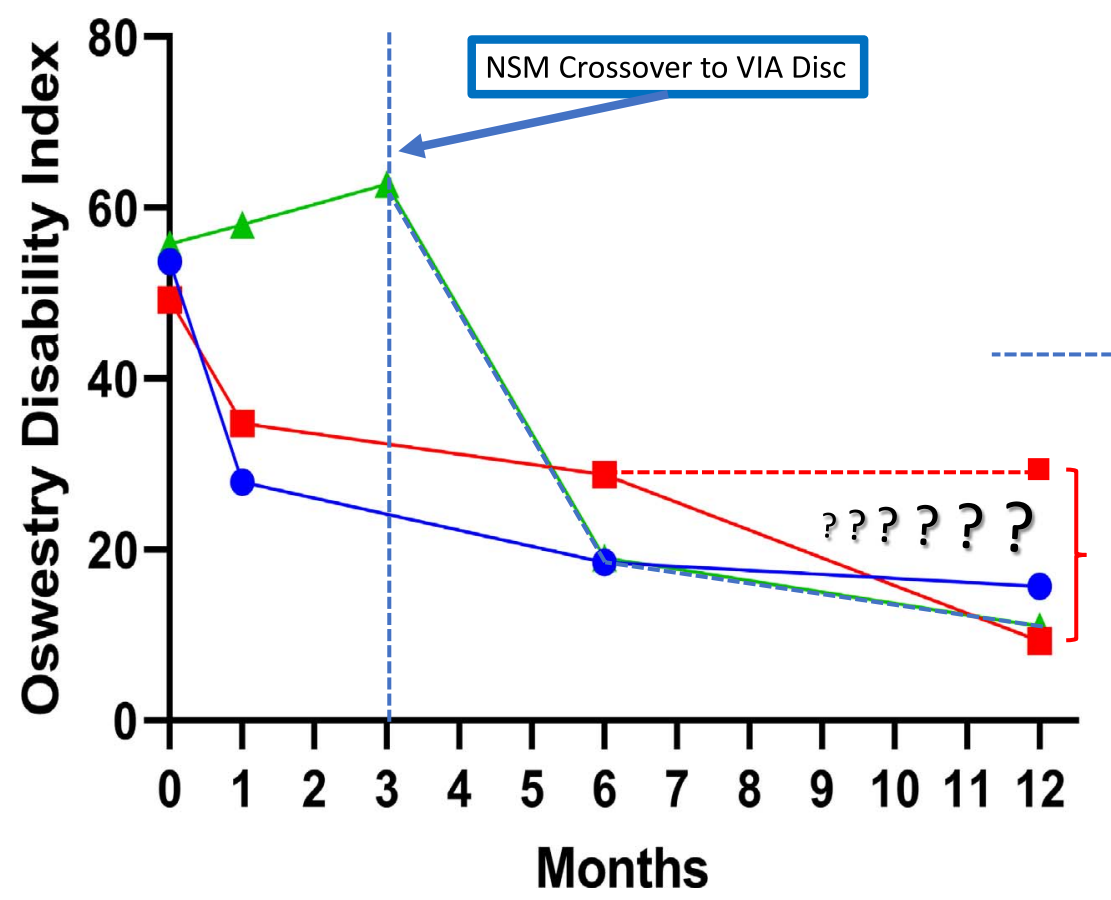

- Active Allograft

$\rightarrow$ Placebo

\pm Conservative Care NSM Crossover

A Placebo Subject Dropped out after 6 months and the trajectory in the small group was skewed. Dotted Line reflects a difference if that subject had been evaluated at 12 months

Figure 5. Functional improvement was evident in both of the active treatment groups in the study. Subjects who had been randomized to continue nonsurgical (NSM) demonstrated no improvement. The NSM subjects were further evaluated at 3 months (vertical dashed line), and unable to continue, all subjects crossed over to VIA Disc Tissue Matrix. Their response followed the trajectory of the active arms of the study and outcome demonstrated the largest improvement. One subject in the saline-treated group could not continue the study, and after 6 months crossed over to VIA Disc. The horizontal dashed line reflects that progress averaged into the small (4 subjects) placebo group. All patients receiving the randomized allograft completed the study in their cohort. In total, 21 of 24 subjects received the VIA Disc Tissue Matrix either randomized or open label. 


\section{6-Month Follow-Up, Random Subject}

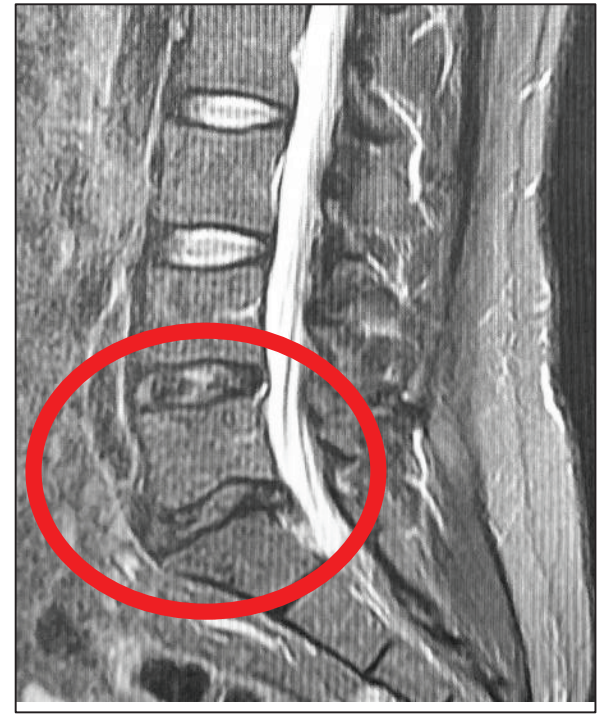

\section{VAST Subject Entry}

Visual Analogue Scale: 55

Oswestry Disability Index: $47 \%$

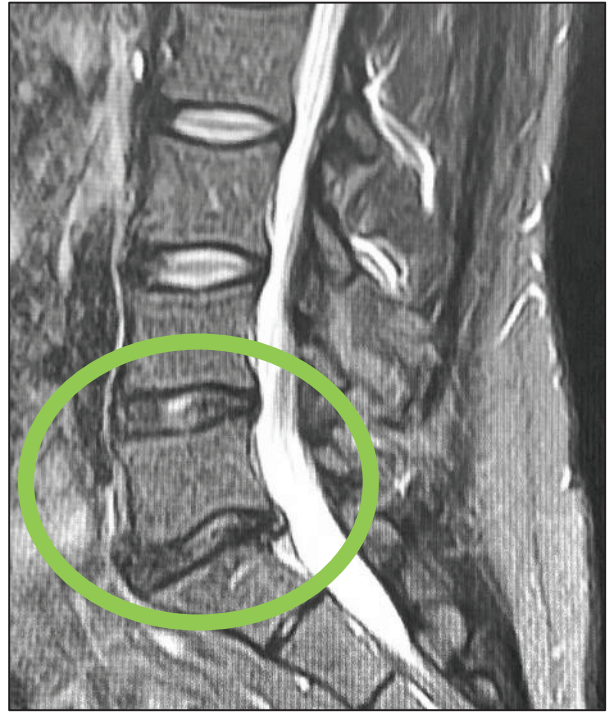

\section{6-Month Post VIA Disc}

Visual Analogue Scale: 17

Oswestry Disability Index: 18\%

Figure 6. MRI images were used to qualify patients for inclusion in the study. Modified Pfirrmann scores between 3 and 6 were acceptable evaluations for participation. Signal intensity and morphologic distinction in the nucleus are noted. More important, pain reduction and functional improvement supported patient satisfaction in the treatment.

\section{2-month Follow-Up, Random Subject}

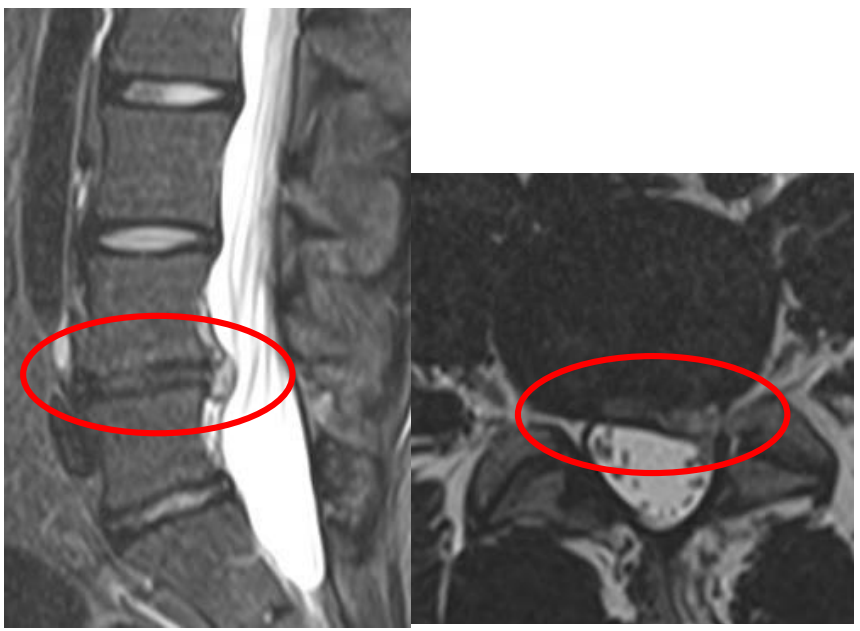

VAST Subject Entry

Visual Analogue Scale: 72

Oswestry Disability Index: $48 \%$

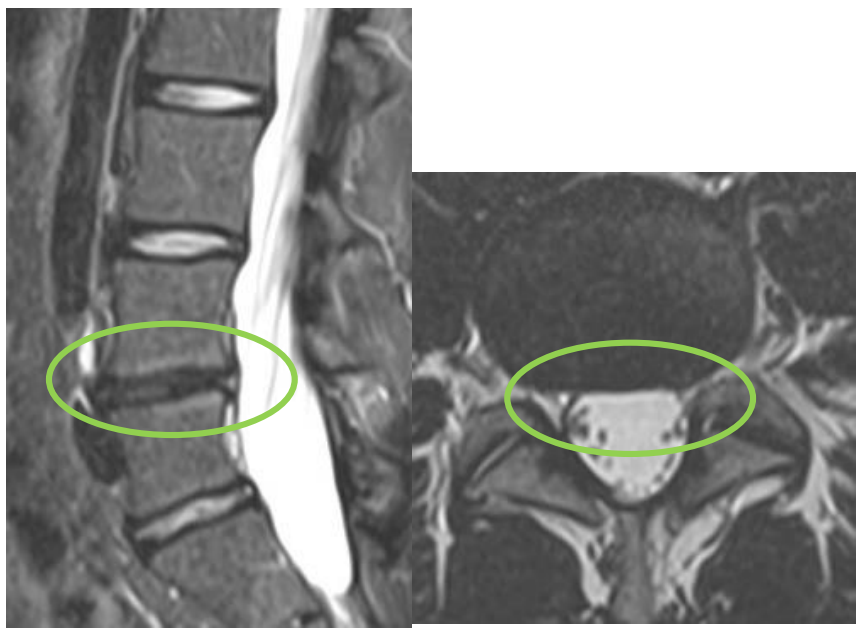

12-Month Post VIA Disc

Visual Analogue Scale: 9

Oswestry Disability Index: $17 \%$

Figure 7. Images at 12 months demonstrated improvement in morphology, disc height, and patient indices of pain and functional improvement followed. 


\section{DISCUSSION}

The high prevalence, incidence, and economic ramifications of degenerative disc disease have guided numerous attempts to intervene with treatments that provide more than palliative solutions to pain. All previous and current human studies of transplanted cells have involved the use of autologous or allogeneic cells requiring ex vivo expansion of the cells. This expansion is costly, time-consuming, and strictly regulated, guiding technology that to date has not provided compelling data for clinical translation. Using allograft preparation technology that has been sufficiently refined to produce allograft that has been minimally manipulated, this study has demonstrated that it is possible to safely offer percutaneous delivery that reduces pain, enhances functional recovery, maintains disc height, and can be administered in a physician's office using sterile technique.

Several key observations emerged from the interim analysis of the VAST Trial. Although conducted with a small population, the 12-month trial provided encouraging evaluations:

1. Adverse events were limited to a small subpopulation within the study and were transitory and not related to the treatments. All events resolved, although a couple of subjects required treatment. The percentages of subjects in the trial were approximately similar, but the small numbers in this safety cohort may not be applicable to larger treatment populations.

2. Supplemental allograft appears to provide an effective resolution of pain, to enhance functional recovery, and to sustain disc height not only at the index level but at levels rostral to the treated level(s) as well.

3. All 16 subjects randomized to and receiving the allografts showed progressive and sustained improvement over the 12 months over which data were collected.

4. Within the cohort randomized to receive saline placebo, 1 of the 4 subjects did not improve and, beset by unremitting pain, crossed over and was treated with VIA Disc Matrix in an open-label manner.

5. None of the subjects randomized to NSM were able to complete the study in their cohort. All 4 subjects crossed over to receive the VIA Disc Matrix after an additional 3 months of NSM and demonstrated immediate and marked improvement in both pain relief and functional improvement that was sustained through the 12-month evaluation.

6. Meaningful clinical improvement was achieved in the cohort randomized to receive VIA Disc Matrix by 6 months and was maintained over the course of the study, attaining a $71 \%$ reduction at 6 months that reached $78 \%$ by 12 months. Those subjects receiving placebo were only able to achieve a $26 \%$ reduction at 6 months. Conservative-care subjects crossed over to open-label VIA Disc at 3 months, and by 6 months had attained $88 \%$ reduction in pain, which at 12 months was $89 \%$. Note that 1 of the subjects receiving the placebo could not continue to 12 months, and the remaining 3 did achieve $65 \%$ reduction but from a population reduced and selected by the loss of a subject due to intolerable pain.

7. Meaningful clinical improvement as measured by ODI was achievable by 6 months and maintained over the course of the study. Subjects randomized to VIA Disc achieved 35-point improvement by 6 months that improved by 45.67 points at 12 months. The placebo group achieved a 20.51-point improvement at 6 months and, despite the dropout of 1 subject, achieved 39.92 points at 12 months. Subjects originally randomized to continue conservative care did not fare well until crossing over and following the openlabel VIA Disc placement, achieved 43.35point improvement by 6 months that further improved to 51.75 points by 12 months.

8. Subjects who crossed over and were aware of receiving the supplemental allograft demonstrated the largest net gains and the fewest AEs in the study.

The critical component of safety was demonstrated in this 24-subject interim analysis of the VAST Trial data. Separate from safety, the enrolled subjects demonstrated meaningful clinical improvement and disc height sustenance. The supplementation of the disc with viable allograft was able to achieve a marked reduction in pain, an improvement of function, and a resolution of symptoms in degenerated discs detect by MR imaging, which was encouraging. The context and connection of image and symptoms has been defined several times, notably demonstrating that imaging alone is an insufficient index to evaluate clinical symptoms. ${ }^{52,53}$ 
Given the durability of the allograft over the 12 months of the study, it will be interesting to evaluate the data from the larger group to see whether the results from the first 24 patients are representative of the entire population studied. The key comparison will be in the improvement noted in the symptomatic discs, which differs significantly from imaging studies that evaluate disc degeneration in asymptomatic patients. Being able to show a comparable improvement in anatomical evaluation in concert with the remission of pain offers unique information that could help to identify the appropriate patients who may benefit from the treatment. Treatments that avoid care or mask symptoms without improving the condition do little for the patient or the health treatment community. By using a supplemental allograft that retains viable components of healthy disc, this treatment combines the expectation of mechanical support with biochemical process to promote disc healing while providing structural support. Being able to reduce disability, enhance activity, and partially preserve spinal motion provides a meaningful clinical intervention that has been shown to be safe and, in this small population, effective.

Whereas the mechanism of action remains to be fully elucidated, many studies have suggested that a variety of viable cells transplanted into the disc can generate extracellular matrix and provide the necessary cellular components required to prevent further disc degeneration following discectomy. As noted in the introduction, cell therapy alone has not shown itself to provide sufficient intervention with regard to either efficacy or duration. The opportunity to pair a disc tissue matrix with cells may provide a supplemental allograft to supplement the lost tissue and cells consequent to the degenerative process. Although it is impossible to fully know whether the cells can differentiate and survive or produce signaling agents that cause the intervertebral disc to heal, this study does confirm that this allograft technology can be administered in a controlled, safe, and regulated environment and that it has produced positive provisional clinical results.

\section{REFERENCES}

1. Spine: low back and neck pain. In: Jacobs JJ, Andersson GB, Bell J-E, Weinstein SL, et al, eds. United State Bone and Joint Initiative: The Burden of Musculoskeletal Disease in the United States. 2nd ed. Rosemont, IL: American Academy of Orthopaedic Surgeons; 2011:21e56.

2. DePalma MJ, Ketchum JM, Saullo T. What is the source of chronic lower back pain and does age play a role? Pain Med. 2011;12(2):224-233. doi:10.1111/j.1526-4637.2010.01045.x

3. Kelsey J, White AA. Epidemiology and impact of low back pain. Spine. 1980;6:133-142. doi:10.1097/00007632-19800300000007

4. Waddell G. A new clinical model for the treatment of low back pain. Spine. 1987;12:632-644. doi:10.1097/00007632198709000-00002

5. Andersson GBJ. The epidemiology of spinal disorders. In: Frymoyer JW, ed. The Adult Spine: Principles and Practice. Philadelphia, PA: Lippincott-Raven; 1997:93-141.

6. Dieleman JL, Baral R, Birger M, et al. US spending on personal health care and public health, 1996-2013. JAMA. 2016;316(24):2627-2646. doi:10.1001/jama.2016.16885

7. Kraemer J. Bandscheibenbedingte Erkrankungen. 4th ed. Stuttgart, Germany: G. Thieme; 1997.

8. Global Burden of Disease Study 2013 Collaborators. Global, regional, and national incidence, prevalence, and years lived with disability for 301 acute and chronic diseases and injuries in 188 countries, 1990-2013: a systematic analysis for the Global Burden of Disease Study 2013. Lancet. 2015;386:743-800.

9. North American Spine Society Task Force on Clinical Guidelines. Phase III Clinical Guidelines for Multidisciplinary Spine Care Specialists. Spondylosis, Lytic Spondylolisthesis, and Degenerative Spondyloslisthesis (SLD). La Grange, IL: North American Spine Society; 2000.

10. Gometz A, Maislen D, Youtz C, et al. The effectiveness of prehabilitation (prehab) in both functional and economic outcomes following spinal surgery: a systematic review. Cureus. 2018;10(5):e2675. Published May 23, 2018. doi:10.7759/cureus. 2675

11. Thompson JP, Pearce RH, Schechter MT, Adams ME, Tsang IK, Bishop PB. Preliminary evaluation of a scheme for grading the gross morphology of the human intervertebral disc. Spine. 1990;15(5):411-415. doi:10.1097/00007632-19900500000012

12. Kettler A, Wilke HJ. Review of existing grading systems for cervical or lumbar disc and facet joint degeneration [published correction appears in Eur Spine J. 2006;15(6):705718]. doi:10.1007/s00586-005-0954-y

13. Antoniou J, Steffen T, Nelson F, et al. The human lumbar intervertebral disc: evidence for changes in the biosynthesis and denaturation of the extracellular matrix with growth, maturation, ageing, and degeneration. J Clin Invest. 1996;98:996-1003. doi:10.1172/JCI118884

14. MacIntosh JE, Bogduk N. The morphology of the lumbar erector spinae. Spine. 1987;12(7):658-668. doi:10.1097 00007632-198709000-00004

15. Parke WW, Watanabe R. Lumbosacral intersegmental epi-spinal axons and ectopic ventral nerve rootlets. $J$ Neurosurg. 1987;7:269-277. doi:10.3171/jns.1987.67.2.0269

16. Rydevik R, Brown MD. Lundborg, G. Pathoanatomy and pathophysiology of nerve root compression. Spine. 1984;9:7-15. doi:10.1097/00007632-198401000-00004

17. Watanabe R, Parke WW. Vascular and neural pathology of lumbosacral spinal stenosis. J Neurosurg. 1986;64;1:64-70. doi:10.3171/jns.1986.64.1.0064

18. Lyons G, Eisenstein SM, Sweet MB. Biochemical changes in intervertebral disc degeneration. Biochim Biophys Acta. 1981;673(4):443-53. doi:10.1016/0304-4165(81)90476-1 
19. Urban JP, Roberts S. Degeneration of the intervertebral disc. Arthritis Res Ther. 2003;5:120-130. doi:10.1186/ar629

20. Roberts S, Menage J, Duance V, Wotton S, Ayad S. 1991 Volvo Award in basic sciences. Collagen types around the cells of the intervertebral disc and cartilage end plate: an immunolocalization study. Spine. 1991;16;9:1030-1038.

21. Urban JP, Holm S, Maroudas A. Diffusion of small solutes into the intervertebral disc: an in vivo study. Biorheology. 1978;15:203-221. doi:10.3233/bir-1978-153-409

22. Kuettner KE, Pauli BU, Gall G, Memoli VA, Schenk RK. Synthesis of cartilage matrix by mammalian chondrocytes in vitro. I. Isolation, culture characteristics, and morphology. $J$ Cell Biol. 1982;93:743-750. doi:10.1083/jcb.93.3.743

23. Buckwalter JA. Aging and degeneration of the human intervertebral disc. Spine. 1995;20:1307-1314. doi:10.1097/ 00007632-199506000-00022

24. Boos N, Weissbach S, Rohrbach H, Weiler C, Spratt KF, Nerlich AG. Classification of age-related changes in lumbar intervertebral discs: 2002 Volvo Award in basic science. Spine. 2002;27:2631-2644. doi:10.1097/00007632-200212010-00002

25. Buttner-Janz, K. The Development of the Artificial Disc SB Charité. Dallas, TX: Hundley \& Associates; 1992.

26. Buttner-Janz K, Schellnack K, Zippel H. Biomechanics of the SB Charité lumbar intervertebral disc endoprosthesis. Int Orthop. 1989;13(3):173-176. doi:10.1007/bf00268042

27. Griffith SL, Shelokov AP, Buttner-Janz K, et al. A multicenter retrospective study of the clinical results of the LINK SB Charité intervertebral prosthesis: the initial European experience. Spine. 1994;19:1842-1849. doi:10.1097/00007632199408150-00009

28. Lemaire JP, Skalli W, Lavaste F, et al. Intervertebral disc prosthesis: results and prospects for the year 2000. Clin Orthop. 1997;337:64-76. doi:10.1097/00003086-199704000-00009

29. Ray CD. The artificial disc. In: Weinstein JN, ed. Clinical Efficacy and Outcome in the Diagnosis and Treatment of Low Back Pain. New York, NY: Raven Press; 1992:205-226.

30. Schoenmayr R, Lotz C, Lotz-Metz G, et al. The implantation of a hydrogel disc nucleus prosthesis in the degenerative lumbar disc disease: early experiences with 10 patients. In: Proceedings of the International Intradiscal Therapy Society, 10th Annual MeetingMay 28-31, 1997 Naples, FL.

31. Zeegers WS, Bohnen LM, Laaper M, et al. Artificial disc replacement with the modular type SB Charité III: 2-year results in 50 prospectively studied patients. Eur Spine J. 1999;8:210-217. doi:10.1007/s005860050160

32. An HS, Thonar EJ, Masuda K. Biological repair of intervertebral disc. Spine. 2003;28:S86-S92. doi:10.1097/01. BRS.0000076904.99434.40

33. Masuda K, Oegema TR Jr, An HS. Growth factors and treatment of intervertebral disc degeneration. Spine. 2004;29:2757-2769. doi:10.1097/01.brs.0000146048.14946.af

34. Wolff JA, Malone RW, Williams P, et al. Direct gene transfer into mouse muscle in vivo. Science. 247:1465-1468. doi:10.1126/science. 1690918

35. Moon SH, Gilbertson LG, Nishida K, et al. Human intervertebral disc cells are genetically modifiable by adenovirus-mediated gene transfer: implications for the clinical management of intervertebral disc disorders. Spine. 2000;25:2573-2579. doi:10.1097/00007632-200010150-00006

36. Ganey TM, Libera J, Moos V, et al. Disc chondrocyte transplantation in a canine model: a treatment for degenerated or damaged intervertebral disc. Spine. 2003; 28:2609-2620. doi:10.1097/01.BRS.0000097891.63063.78

37. Meisel HJ, Siodla V, Ganey T, Minkus Y, Hutton WC, Alasevic OJ. Clinical experience in cell-based therapeutics: disc chondrocyte transplantation. A treatment for degenerated or damaged intervertebral disc. Biomol Eng. 2007;24:5-21. doi:10. 1016/j.bioeng.2006.07.002

38. Krupkova O, Cambria E, Besse L, Besse A, Bowles R, Wuertz-Kozak K. The potential of CRISPR/Cas9 genome editing for the study and treatment of intervertebral disc pathologies. JOR Spine. 2018;1:e1003. https://doi.org/10.1002/ jsp2.1003

39. Moon SH, Nishida K, Gilbertson LG, et al. Biologic response of human intervertebral disc cells to gene therapy cocktail. Spine. 2008;33:1850-1855. doi:10.1097/BRS. 0b013e31817e1cd7

40. Ren S, Liu Y, Ma J, et al. Treatment of rabbit intervertebral disc degeneration with co-transfection by adeno-associated virus-mediated SOX9 and osteogenic protein-1 double genes in vivo. Int J Mol Med. 2013;32:1063-1068. doi:10.3892/ijmm.2013.1497

41. Vadala G, Russo F, Di Martino A, Denaro V. Intervertebral disc regeneration: from the degenerative cascade to molecular therapy and tissue engineering. J Tissue Eng Regen Med. 2015; 9:679-690. doi:10.1002/term.1719

42. Kusior LJ, Vacanti CA, Bayley JC, et al. Tissue engineering of nucleus pulposus in nude mice. Trans Orthop Res Soc. 1999;24:807.

43. Nishida K, Kang JD, Gilbertson LG, et al. Modulation of the biologic activity of the rabbit intervertebral disc by gene therapy: an in vivo study of adenovirus-mediated transfer of the human transforming growth factor $B 1$ encoding gene. Spine. 1999;24:2419-2425. doi:10.1097/00007632-199912010-00002

44. Takegami K, Masuda K, Kumano F, et al. Osteogenic protein-1 is most effective in stimulating nucleus pulposus and annulus fibrosus cells to repair their matrix after chrondroitinase ABC-induced chemonucleolysis. Trans Orthop Res Soc. 1999;24:201.

45. Krishnamoorthy $\mathrm{S}$, Noorani B, Xu C. Effects of encapsulated cells on the physical-mechanical properties and microstructure of gelatin methacrylate hydrogels. Int $\mathrm{J} \mathrm{Mol} \mathrm{Sci}$. 2019;20:5061. doi:10.3390/ijms20205061

46. Zhu Q, Gao X, Temple HT, Brown MD, Gu W. Simulation of biological therapies for degenerated intervertebral discs. J Orthop Res. 2017;35:147-153. doi:10.1002/jor.23284

47. ClinicalTrials.gov. https://clinicaltrials.gov/ct2/show/ NCT02412735. Accessed February 17, 2019.

48. Hudson-Cook N, Tomes-Nicholson K, Breen AC. A revised Oswestry Disability Questionnaire. In: Roland M, Jenner J, eds. Back Pain: New Approaches to Rehabilitation and Education. Manchester, UK: University Press, 1989:187204.

49. Modic MT, Steinberg PM, Ross JS, et al. Degenerative disk disease: assessment of changes in vertebral body marrow with MR imaging. Radiology. 1988;166:193-199. doi:10.1148/ radiology.166.1.3336678

50. Ross JS, Zepp R, Modic MT. The postoperative lumbar spine: enhanced MR evaluation of the intervertebral disc. Am Soc Neuroradiol. 1996;17:323-331.

51. Mochida J, Nomura T, Toh E, Chiba M. The 
importance of preserving disc structure in surgical approaches to lumbar disc herniation. Spine. 1996;21:1556-1563. doi:10. 1097/00007632-199607010-00014

52. Boden SD, Davis DO, Dina TS, Patronas NJ, Wiesel SW. Abnormal magnetic-resonance scans of the lumbar spine in asymptomatic subjects. J Bone Joint Surg Am. 1990;72A;403408.

53. Brinjikji W, Luetmer PH, Comstock B, et al. Systematic literature review of imaging features of spinal degeneration in asymptomatic populations. AJNR Am J Neuroradiol. 2015;36(4):811-816. doi:10.3174/ajnr.A

Disclosures and COI: Dr Beall reports grants from Medtronic, nonfinancial support from Amendia, other from Lilly, other from Synthes, other from Johnson and Johnson, grants from Vitacare, grants from Ortho Kinematics, other from DFine, other from Bone Support, other from Convatec, other from Spinal Ventures, grants from Zyga, grants from Liventa, grants, nonfinancial support and other from Vexim, grants from Mesoblast, grants, personal fees, nonfinancial support and other from Vivex, outside the submitted work.

Corresponding Author: Douglas Beall, MD, Summit Medical Center, 1800 South Renaissance Blvd, Suite 110, Edmond, OK 73013. Phone: (405) 601-2325; Email: db@clinrad.org.

Published 30 April 2020

This manuscript is generously published free of charge by ISASS, the International Society for the Advancement of Spine Surgery. Copyright (c) 2020 ISASS. To see more or order reprints or permissions, see http://ijssurgery.com. 